\title{
EXTREMALLY DISCONNECTED SPACES
}

\section{DONA PAPERT STRAUSS}

Introduction. By an extremally disconnected space we shall mean a Hausdorff space in which the closure of every open set is open. These spaces are known to possess interesting properties. For example, the extremally disconnected spaces can be characterized among the Hausdorff spaces by the fact that any continuous function mapping a dense subspace of an extremally disconnected space to a compact Hausdorff space, can be extended to the whole space. They can also be characterized among the completely regular spaces by the fact that the lattice of continuous functions mapping an extremally disconnected space to the unit interval is complete (cf. [1], $3 \mathrm{~N}$ and $6 \mathrm{M}$ ).

Stonean spaces, i.e. extremally disconnected compact spaces, have received particular attention. A. Gleason has shown that the Stonean spaces are the projective objects in the category of compact spaces [2]. The purpose of this note is to extend Gleason's result and prove that the extremally disconnected spaces are the projective objects in a wider category.

We shall prove the following theorems. If $X$ is any topological space, there is an extremally disconnected space $E_{X}$ associated with $X$. The points of $E_{X}$ are in fact the convergent ultrafilters of open subsets of $X$. If $X$ is Hausdorff, there is a natural mapping from $E_{X}$ to $X$ which maps every convergent ultrafilter to its limit point. This mapping is closed and compact, i.e. it has the property that the inverse image of any point is compact. It is continuous if and only if $X$ is regular.

Thus, if $X$ is a regular Hausdorff space, the mapping from $E_{X}$ to $X$ is closed, continuous and compact. We shall call a mapping with these properties perfect. By using methods based on Gleason's, we shall show that the extremally disconnected spaces are the projective objects in the category of regular Hausdorff spaces and perfect maps. It will follow that $E_{X}$ is the minimal extremally disconnected space that can be mapped on to $X$ by a perfect mapping.

Preliminaries. Let $X$ be any topological space. A filter of open subsets of $X$ is a nonempty set $F$ of subsets of $X$ with the properties: $\varnothing \notin F ; G_{1}, G_{2} \in F \rightarrow G_{1} \cap G_{2} \in F ; G_{1} \supset G_{2} \in F \rightarrow G_{1} \in F$.

An ultrafilter of open subsets of $X$ is a filter which is maximal.

If $G$ is any open subset of $X, \mathfrak{u}_{G}$ will denote the set of ultrafilters

Received by the editors January 27, 1966. 
of open subsets of $X$ which contain $G . S_{X}$ will denote the space whose points are the ultrafilters of open subsets of $X$, and whose topology is defined by taking all the sets of the form $\mathfrak{u}_{G}$ as a basis for the open sets. $S_{X}$ is a Stonean space. (It is readily seen to be the Stonean space associated with the complete Boolean algebra of regular open subsets of $X$.)

$E_{X}$ will denote the subspace of $S_{X}$ consisting of the convergent ultrafilters of open subsets of $X$, an ultrafilter being convergent if it contains all the open neighbourhoods of a point. $E_{X}$ is an extremally disconnected space, as it is readily seen to be everywhere dense in $S_{X}$. If $G$ is any open subset of $X, V_{G}$ will denote the basic open subset $\mathcal{u}_{G} \cap E_{X}$ of $E_{X}$. It is easy to see that the complement of $V_{G}$ is $\mho_{\bar{G}} c$, where $\bar{G}^{c}$ denotes the complement of $\bar{G}$. Thus $\mathcal{V}_{G}$ is closed as well as open, and the sets of the form $v_{G}$ also form a basis for the closed subsets of $X$.

Lemma 1. Let $X$ be any topological Hausdorff space. Let $f_{X}: E_{X} \rightarrow X$ be the function mapping every convergent ultrafilter of open subsets of $X$ to its limit point. Then $f_{X}$ is closed and compact. Furthermore, $f_{X}$ maps any proper closed subset of $E_{X}$ to a proper subset of $X$.

Proof. Firstly, if $G$ is any open subset of $X$, it is clear that $f_{X}\left(\mathcal{U}_{G}\right)=\bar{G}$.

Suppose that $H$ is a closed subset of $E_{X}$. Then there is a family $\left\{G_{\alpha}\right\}_{\alpha \in A}$ of open subsets of $X$ such that $H=\bigcap_{\alpha \in A} V_{G_{\alpha}}$. We may suppose that $\left\{G_{\alpha}\right\}_{\alpha \in A}$ is filtered downwards. It is easy to see that $f_{X}(H)$ $=\bigcap_{\alpha \in A} \bar{G}_{\alpha}$. As this is a closed subset of $X, f_{X}$ is closed.

Now let $x \in X$. If $f_{\bar{X}}^{-1}\{x\}$ is not compact, there is a family of open subsets $\left\{G_{\beta}\right\}_{\beta \in B}$ of $X$, filtered downwards, such that

but, for any $\beta \in B$,

$$
\overline{f_{X}^{-1}}\{x\} \cap \bigcap_{\beta \in B} \mathcal{V}_{G \beta}=\varnothing,
$$

$$
f_{X}^{-1}\{x\} \cap \mho_{G \beta} \neq \varnothing .
$$

Thus $\varnothing \neq f_{X}\left(f_{\bar{X}}^{-1}\{x\} \cap \mho_{G_{\beta}}\right) \subset\{x\} \cap f_{X}\left(\mathcal{V}_{G_{\beta}}\right)=\{x\} \cap \bar{G}$. Hence $x \in \bar{G}_{\beta}$ for every $\beta \in B$.

It follows that the set of open neighbourhoods of $x$ together with all the sets $G_{\beta}$, has the finite intersection property. It is therefore contained in some ultrafilter $U$ of open subsets of $X$. We have $f_{X}(U)=x$ and thus $U \in f_{X}^{-1}\{x\}$. But $U \in \bigcap_{\beta \in B} \mathcal{V}_{G_{\beta}}$-contradicting our assumption that $f_{X}^{-1}\{x\} \cap \bigcap_{\beta \in B} \mho_{G_{\beta}}=\varnothing$.

Finally, suppose that $F$ is a proper closed subset of $E_{X}$. Then there exists an open subset $G$ of $X$ such that $F \subset \mathcal{V}_{G}$ and $\mathcal{V}_{G} \neq E_{X}$. Thus 
there is a nonempty open subset $H$ of $X$ such that $G \cap H=\varnothing$. It follows that $f_{X}(F) \subset \bar{G} \subset H^{c}$ is a proper subset of $X$.

REMARK. It is easy to see that any image of a Hausdorff space under a closed compact mapping is also Hausdorff. Thus the Hausdorff spaces are precisely the spaces which are images of extremally disconnected spaces under closed compact mappings.

Lemma 2. Let $X$ be a regular Hausdorff space. Then $f_{X}$ is continuous.

Proof. Let $G$ be any open subset of $X$. Then there is a family $\left\{H_{\alpha}\right\}_{\alpha \in A}$ of open subsets of $G$ such that $G=\bigcup_{\alpha \in A} H_{\alpha}$ and $\bar{H} \subset G$ for each $\alpha \in A$. It is easy to see that $f_{X}^{-1}(G)=\bigcup_{\alpha \in A} V_{H_{\alpha}}$, which is an open subset of $E_{X}$. Thus $f_{X}$ is continuous.

Leмма 3. An image of a regular Hausdorff space under a perfect mapping is regular.

Proof. Suppose that $X$ is a regular Hausdorff space and that $f$ is a perfect mapping from $X$ on to a topological space $Y$. Let $y \in Y$ and let $H$ be a neighbourhood of $y$. Since $f^{-1}(H)$ is a neighbourhood of the compact set $f^{-1}\{y\}$, there is an open subset $G$ of $X$ such that $f^{-1}\{y\}$ $\subset G \subset \bar{G} \subset f^{-1}(H)$. Now $\left(f\left(G^{c}\right)\right)^{c}$ is an open set containing $y$. Also, $\left(f\left(G^{c}\right)\right)^{c} \subset f(G)$ and so $\left[\left(f\left(G^{c}\right)\right)^{c}\right]-\subset[f(G)]^{-} \subset f(\bar{G}) \subset H$. This shows that $Y$ is regular.

Remark. From Lemmas 1 and 2 it follows that the regular Hausdorff spaces are precisely the spaces which are images of extremally disconnected spaces under perfect mappings.

Lemma 4. Let $f$ be a closed mapping from a topological space $X$ to a topological space $Y$. If $f^{-1}$ maps points to compact sets, then $f^{-1}$ maps all compact sets to compact sets. This shows that the composite of two perfect mappings is perfect, and that we may speak of the category of regular Hausdorff spaces and perfect mappings.

Proof. Let $C$ be a compact subset of $Y$. Suppose that there is a family $\left\{H_{\alpha}\right\}_{\alpha \in A}$ of closed subsets of $X$ such that $\left\{H_{\alpha}\right\}_{\alpha \in A}$ is filtered downwards, $f^{-1}(C) \cap \bigcap_{\alpha \in A} H_{\alpha}=\varnothing$ but that, for any $\alpha \in A, f^{-1}(C)$ $\cap H_{\alpha} \neq \varnothing$. Since $f\left(H_{\alpha}\right)$ is a closed set which meets $C$, there exists a point $y \in C$ such that $y \in \bigcap_{\alpha \in A} f\left(H_{\alpha}\right)$. For every $\alpha \in A, f^{-1}\{y\}$ $\cap H_{\alpha} \neq \varnothing$. Since $f^{-1}\{y\}$ is compact, there exists a point $x \in f^{-1}\{y\}$ $\cap \bigcap_{\alpha \in A} H_{\alpha}$. But $x \in f^{-1}(C)$-contradicting our assumption that $f^{-1}(C) \cap \bigcap_{\alpha \in A} H_{\alpha} \neq \varnothing$.

Lemma 5. Let $X$ and $Y$ be topological spaces and let $f$ be a compact mapping from $X$ onto $Y$. Then there is a closed subspace $F$ of $X$ such 
that $f(F)=Y$ and such that no proper closed subspace of $F$ has this property.

Proof. Let $\Gamma$ be any simply ordered family of closed subsets of $X$ which are mapped onto $Y$ by $f$. Then $F=\bigcap_{H \in \Gamma} H$ is closed. Also, for any $y \in Y$ and $H \in \Gamma, f^{-1}\{y\} \cap H \neq \varnothing$. Since $f^{-1}\{y\}$ is compact, it follows that $f^{-1}\{y\} \cap F \neq \varnothing$, and hence that $f(F)=Y$.

Lemma 5 now follows from Zorn's lemma.

The following results are based on [2].

Lemma 6. Suppose that $f$ is a closed continuous mapping from a topological space $Y$ onto a topological space E. Suppose also that $f$ does not map any proper closed subset of $Y$ onto $E$. Then, if $H$ is any open subset of $Y, f(H) \subset\left[\left(f\left(H^{c}\right)\right)^{c}\right]-$.

Proof. Consider the open subset $W=H \cap\left(f^{-1}\left[\left(f\left(H^{c}\right)\right)^{c}\right]^{-}\right)^{c}$ of $Y$. If $y \in W$, then $f(y) \notin\left(f\left(H^{c}\right)\right)^{c}$. Thus $f(y) \in f\left(H^{c}\right)$, and there exists $z \in H^{c}$ such that $f(z)=f(y)$. But $z \notin W$. This shows that $f(W) \subset f\left(W^{c}\right)$ and that $f\left(W^{c}\right)=E$. It follows that $W^{c}=Y$ and that $W=\varnothing$.

Lemma 7. Suppose that $f$ is a closed continuous mapping of a topological Hausdorff space $Y$ onto an extremally disconnected space $E$. If $f$ does not map any proper closed subset of $Y$ onto $E, f$ is a homeomorphism.

Proof. We must show that $f$ is one-one. Suppose then that $y$ and $z$ are distinct points of $Y$ for which $f(y)=f(z)=e$, say. There are two disjoint open subsets $G$ and $H$ of $Y$ such that $y \in G$ and $z \in H$. The open subsets $\left(f\left(G^{c}\right)\right)^{c}$ and $\left(f\left(H^{c}\right)\right)^{c}$ of $E$ are disjoint, and, since $E$ is extremally disconnected, their closures are disjoint. But $e \in f(G)$ $\cap f(H) \subset\left[\left(f\left(G^{c}\right)\right)^{c}\right]-\cap\left[\left(f\left(H^{c}\right)\right)^{c}\right]-$, contradicting our last statement.

THEOREM. In the category of regular Hausdorff spaces and perfect mappings, the extremally disconnected spaces are projective objects.

Proof. Suppose that $f$ is a perfect mapping from a regular Hausdorff $Y$ on to a regular Hausdorff space $X$, and that $g$ is a perfect mapping from an extremally disconnected space $E$ into $X$. We shall show that there is a perfect mapping $h$ from $E$ to $Y$ for which the following diagram commutes:

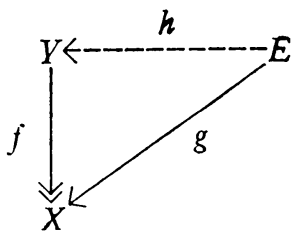


Consider the subset $P=\{(e, y): g(e)=f(y)\}$ of $E \times Y$. We shall first show that the projection $\pi_{1}$ of $P$ on to $E$ is perfect. It is clearly continuous and, as $\pi_{1}^{-1}\{e\}=\{e\} \times f^{-1}\{g(e)\}$, it is clearly compact. We must show that it is closed. Let $F$ be a closed subset of $P$ and let $e \in E-\pi_{1}(F)$. Then the compact set $\{e\} \times f^{-1}\{g(e)\}$ and the closed set $F$ are disjoint subsets of $E \times Y$. There is thus an open set $H$ in $E \times Y$ such that $\{e\} \times f^{-1}\{g(e)\} \subset H$ and $F \cap H=\varnothing$. And we may further suppose that, for some integer $n, H$ can be written as $H$ $=\mathrm{U}_{i=1}^{n}\left(D_{i} \times W_{i}\right)$ where $D_{i}$ is an open subset of $E$ and $W_{i}$ is an open subset of $Y$ for each $i=1, \cdots, n$. Put $D=\bigcap_{i=1}^{n} D_{i}$ and $W=\bigcup_{i=1}^{n} W_{i}$. Then $\{e\} \times f^{-1}\{g(e)\} \subset D \times W$ and $F \cap(D \times W)=\varnothing$. Now put $B=D \cap g^{-1}\left(\left(f\left(W^{c}\right)\right)^{c}\right)$. Then $B$ is an open set containing $e$. Furthermore, for any $b \in B, f^{-1}\{g(b)\} \subset W$ and so $F \cap \pi_{1}^{-1}\{b\}=\varnothing$. This shows that $B \cap \pi_{1}(F)=\varnothing$, that $\pi_{1}(F)$ is closed and that $\pi_{1}$ is a closed mapping.

Similarly, the projection $\pi_{2}$ of $E \times Y$ onto $Y$ is perfect.

Now, by Lemma 5 , there is a closed subset $P_{0}$ of $P$ for which $\pi_{1}\left(P_{0}\right)=E$ and which is minimal subject to this condition. By Lemma $7, \pi_{1} / P_{0}$ is a homeomorphism and has an inverse, say $\sigma$. Finally, if we put $h=\pi_{2} \sigma, h$ will be the required mapping.

REMARKs. That the extremally disconnected spaces are precisely the projective objects in the category that we are considering, follows from a theorem in [2] which implies that any projective object in our category is extremally disconnected.

It follows from the above theorem and Lemma 7 that $E_{X}$ is the only extremally disconnected space which can be mapped on to $X$ by a perfect mapping which does not map any proper closed subset on to $X$. It also follows that any extremally disconnected space which can be mapped on to $X$ by a perfect mapping contains a homeomorphic image of $E_{X}$.

$X$ and $E_{X}$ have many topological properties in common. If $X$ is a regular Hausdorff space, it is fairly easy to see that the following statements hold. $E_{X}$ is compact if and only if $X$ is; $E_{X}$ is paracompact if and only if $X$ is; $E_{X}$ is locally compact if and only if $X$ is; $E_{X}$ is Lindelöf if and only if $X$ is; and $X$ is normal if $E_{X}$ is. I have not been able to determine whether the normality of $X$ implies that of $E_{X}$.

\section{REFERENCES}

1. L. Gillman and M. Jerison, Rings of continuous functions, Van Nostrand, 1960.

2. A. Gleason, Projective topological spaces, Illinois J. Math. 2 (1958), 482-489.

3. M. H. Stone, Applications of the theory of Boolean rings to general topology, Trans. Amer. Math. Soc. 41 (1937), 375-481. 